(C) 2014 IEEE. Personal use of this material is permitted. Permission from IEEE must be obtained for all other uses, in any current or future media, including reprinting/republishing this material for advertising or promotional purposes, creating new collective works, for resale or redistribution to servers or lists, or reuse of any copyrighted component of this work in other works. 


\section{Control of a Single-Switch Two-Input Buck Converter for MPPT of Two PV Strings}

\author{
Andoni Urtasun \\ Department of Electrical and Electronic Engineering \\ Public University of Navarra (UPNA) \\ Pamplona, Spain \\ andoni.urtasun@unavarra.es
}

\author{
Dylan Dah-Chuan Lu \\ School of Electrical and Information Engineering \\ The University of Sydney \\ NSW 2006, Australia \\ dylan.lu@sydney.edu.au
}

\begin{abstract}
In this paper, the configuration composed by a TwoInput Buck (TIBuck) converter and a boost inverter is proposed for low-voltage grid-connected PV systems. This configuration is attractive for this application because it has high efficiency and can achieve dual maximum power point tracking (MPPT) with only one active switch. However, in this system, the nonlinear characteristics of the converter and the two PV arrays complicate the control. By means of a smallsignal modeling, the control theme of the two PV voltages is formulated and the effect of the nonlinearities is presented. Simulation results are reported to validate the theoretical analysis, showing the dual MPPT capability.
\end{abstract}

\section{INTRODUCTION}

Photovoltaic systems are experiencing continuous expansion and development, particularly in grid-connected applications. More than $39 \mathrm{GW}$ were added during 2013, bringing worldwide total capacity to approximately $139 \mathrm{GW}$. Almost half of all PV capacity in operation was added in the past two years, and $98 \%$ has been installed since the beginning of 2004 [1].

An important fraction of the total capacity corresponds to residential rooftop installations. These systems are often of low-power and low-voltage, requiring a step-up conversion in order to be connected to the electric grid. A simple and reliable solution is to install a boost inverter between the PV generator and the grid, as shown in Fig. 1(a) [2], [3]. This configuration is attractive from a component count perspective but it only performs one MPPT algorithm per converter. However, given that different PV module orientations and shading conditions are common in this application, the single boost inverter can result in significant power losses [4].

An attractive alternative is a two-stage boost inverter, where the first stage is a boost converter and the second stage is an H-bridge inverter [5]. In order to perform two different MPPTs, two dc/dc boost converters can be placed in parallel, as shown in Fig. 1(b) [6]. Although the conversion efficiency is lower when compared to the previous configuration, the overall efficiency is higher for rooftop applications under partial shading condition, thanks to the dual MPPT capability.

This paper proposes a new configuration where the first stage is a Two-Input Buck (TIBuck) converter and the second stage is a boost inverter, as shown in Fig. 1(c). Similar to the previous case, this configuration also achieves two MPPTs. However, only one active switch is required in the proposed scheme as compared to Fig. 1(a), making the system more cost-effective and reliable. Furthermore, given that the switch and the diode have low voltage stress, the TIBuck conversion efficiency is very high [7]. The overall conversion efficiency is expected to be slightly lower than the single-boost inverter due to the additional TIBuck stage. However, the proposed configuration will improve MPPT efficiency for rooftop applications under different shading conditions.
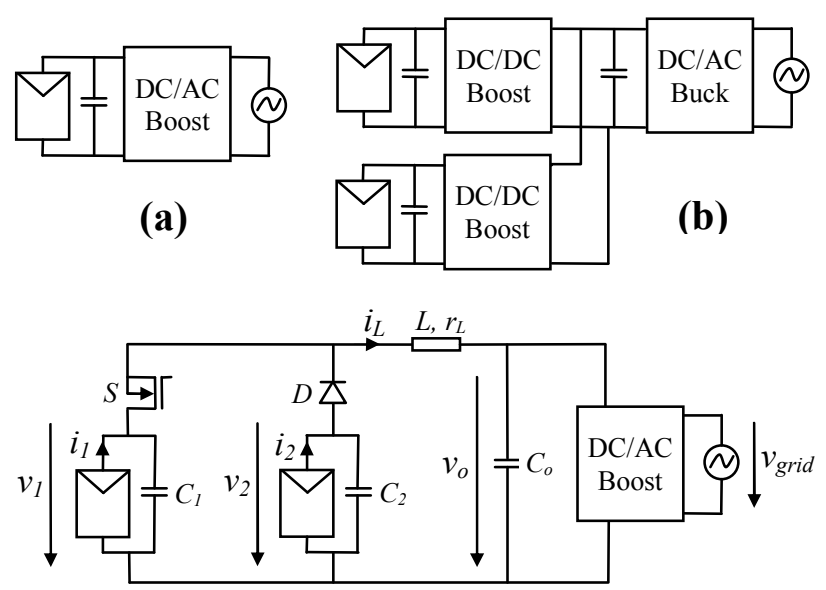

(c)

Figure 1. Configurations for low-voltage grid-connected PV systems: (a) Single boost inverter, (b) Multiple boost converters, (c) Proposed scheme.

Note that the TIBuck converter was originally proposed by Sebastian et al. to improve ac/dc conversion efficiency, 
and the output voltage was to be controlled [7]. This paper, however, is concerned about the dual MPPT and deals with the two input voltage regulation. The nonlinearity of the two PV arrays must be considered, which adds complexity to the analysis of the nonlinear converter [5], [8]-[10]. Smallsignal modeling is carried out in order to apply linear control techniques.

The paper is organized as follows. In Section II, the control scheme for the proposed configuration is presented. The small-signal model is then derived in Section III. The regulation of both input voltages is presented in Section IV. Then, in Section V, simulation results are provided to verify the control performance. Finally, conclusions of this work are given in Section VI.

\section{DUAL MPPT WITH TIBUCK CONVERTER}

The proposed configuration is shown in Fig. 1(c), where $i_{1}$ and $i_{2}$ are the PV currents, $v_{1}$ and $v_{2}$ are the PV voltages, $v_{o}$ is the output voltage, $i_{L}$ is the inductor current, and $S$ is the commutation function ( 0 for off state or 1 for on state). The first stage is similar to the conventional buck converter, excluding that a second input is added, becoming a TIBuck converter. Two capacitors, $C_{1}$ and $C_{2}$, are placed in parallel with the two PV strings respectively to change the causality from current source to voltage source and reduce the voltage ripple.

The elements used throughout the paper are presented in Table I and II. Table I shows the features of the TIBuck converter. The capacitor values have been chosen so that the MPPT losses due to the voltage ripple are lower than $0.2 \%$ according to [11]. The inductor value is obtained in order to avoid discontinuous conduction mode and limit the current ripple. The equivalent resistance includes the inductor, switch and diode conduction losses. Table II shows the specifications of the PV arrays. The TIBuck converter makes it possible to interface with different types of PV modules in its two inputs, with the only restriction that $v_{l}$ must be higher than $v_{2}$. For this reason, three polycrystalline modules have been connected in series at input 1 and two monocrystalline modules have been connected in series at input 2 .

The control scheme is shown in Fig. 2. The MPPT algorithm requires the measured variables $v_{1, m}, v_{2, m}, v_{o, m}$, and $i_{L, m}$ as inputs. With this information, it provides the reference voltages $v_{1, \text { ref }}$ and $v_{2, \text { ref }}$ to be controlled. In some situations, the PV power does not have to be maximized and needs to be limited [12]. In any case, this paper deals with the voltage regulation, and not with the MPPT algorithm or power limitation. It is thus assumed that the reference voltages are known.

The two input voltages can be controlled by the two degrees of freedom, namely the TIBuck switch commutation and the output voltage $v_{o}$. From the PV1 voltage error, the control obtains the switch duty cycle reference, $d_{\text {ref }}$, from which the switch commutations are found after the modulation. From the PV2 voltage error, the other control obtains the output voltage reference, $v_{o, r e f}$. In turn, this voltage is then regulated by the inverter. The output voltage control is dynamically restricted to around $20 \mathrm{~Hz}$ due to the $100 \mathrm{~Hz}$ ripple present in single-phase inverters. As it will be shown later, the slow inner voltage control will hamper the PV2 voltage regulation. On the other hand, the voltage $v_{o}$ only requires slight variation, resulting in small impact on the boost inverter rated operation.

TABLE I. FEATURES OF THE TIBUCK CONVERTER

\begin{tabular}{|c|c|}
\hline Rated power & $400 \mathrm{~W}$ \\
\hline Input capacitor $C_{I}$ & $30 \mu \mathrm{F}$ \\
\hline Input capacitor $C_{2}$ & $30 \mu \mathrm{F}$ \\
\hline Inductor $L$ & $40 \mu \mathrm{H}$ \\
\hline Equivalent resistance $r_{L}$ & $65 \mathrm{~m} \Omega$ \\
\hline Rated output voltage $V_{0}$ & $40 \mathrm{~V}$ \\
\hline Commutation frequency $f$ & $50 \mathrm{kHz}$ \\
\hline
\end{tabular}

TABLE II. SPECIFICATIONS OF THE PV ARRAYS

\begin{tabular}{|c|c|}
\hline PV1 module model & Sharp NE-080T1J \\
\hline PV1 array MPP power $P_{M P P 1}$ & $240 \mathrm{~W}$ \\
\hline PV1 array MPP voltage $V_{M P P 1}$ & $51.9 \mathrm{~V}$ \\
\hline PV1 array MPP current $I_{M P P 1}$ & $4.63 \mathrm{~A}$ \\
\hline PV1 array open-circuit voltage $V_{o c 1}$ & $64.8 \mathrm{~V}$ \\
\hline PV1 array short-circuit current $I_{S C 1}$ & $5.15 \mathrm{~A}$ \\
\hline PV2 module model & Hurricane HS-80D \\
\hline PV2 array MPP power $P_{M P P 2}$ & $160 \mathrm{~W}$ \\
\hline PV2 array MPP voltage $V_{M P P 2}$ & $36 \mathrm{~V}$ \\
\hline PV2 array MPP current $I_{M P P 2}$ & $4.5 \mathrm{~A}$ \\
\hline PV2 array open-circuit voltage $V_{o c 2}$ & $44 \mathrm{~V}$ \\
\hline PV2 array short-circuit current $I_{S C 2}$ & $4.7 \mathrm{~A}$ \\
\hline
\end{tabular}

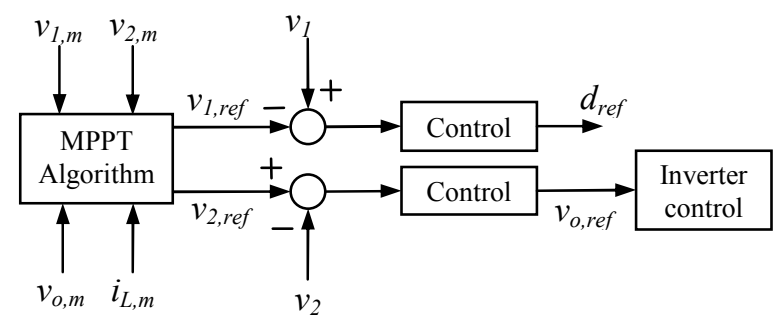

Figure 2. Control scheme for dual MPPT with TIBuck converter.

\section{SMALL-SignAL MODELING}

Since the output voltage is controlled by the boost inverter, it will be considered as a controlled voltage source. It is also assumed that the TIBuck converter is operating in continuous conduction mode. In this mode, the switch is conducting and the diode is off for $S=1$, while the switch is off and the diode is conducting for $S=0$. From Kirchhoff's laws applied to the system presented in Fig. 1(c), and considering average values, one obtains

$$
\begin{gathered}
C_{1} \cdot \frac{d v_{1}}{d t}=i_{1}-d \cdot i_{L} \\
C_{2} \cdot \frac{d v_{2}}{d t}=i_{2}-(1-d) \cdot i_{L} \\
L \cdot \frac{d i_{L}}{d t}+r_{L} \cdot i_{L}=d \cdot v_{1}+(1-d) \cdot v_{2}-v_{0},
\end{gathered}
$$


where $d$ is the TIBuck switch duty cycle.

From (1)-(3), the steady-state equations can be worked out as

$$
\begin{gathered}
I_{1}=D \cdot I_{L} \\
I_{2}=(1-D) \cdot I_{L} \\
V_{0}=D \cdot V_{1}+(1-D) \cdot V_{2}-r_{L} \cdot I_{L},
\end{gathered}
$$

where steady-state variables are expressed in capital letters.

The converter model represented by (1)-(3) is nonlinear. In order to use linear control techniques, small-signal analysis is applied to those equations, resulting in

$$
\begin{gathered}
C_{1} \cdot \frac{d \hat{v}_{1}}{d t}=\hat{i}_{1}-D \cdot \hat{i}_{L}-I_{L} \cdot \hat{d} \\
C_{2} \cdot \frac{d \hat{v}_{2}}{d t}=\hat{i}_{2}-(1-D) \cdot \hat{i}_{L}+I_{L} \cdot \hat{d} \\
L \cdot \frac{d \hat{i}_{L}}{d t}+r_{L} \cdot \hat{i}_{L}=D \cdot \hat{v}_{1}+(1-D) \cdot \hat{v}_{2}+\left(V_{1}-V_{2}\right) \cdot \hat{d}-\hat{v}_{0},
\end{gathered}
$$

where small-signal variables are marked with a circumflex and the operation point is defined by (4)-(6).

The PV current $i_{1} / i_{2}$ depends on the PV voltage, the irradiation and the array temperature through a nonlinear expression. Since the temperature variation is very slow, its small-signal effect can be neglected. The PV array smallsignal model can then be expressed as follows [5]:

$$
\begin{gathered}
\hat{i_{1}}=K_{g 1} \cdot \hat{g}_{1}-\frac{\hat{v}_{1}}{R_{1}} \\
\hat{i}_{2}=K_{g 2} \cdot \hat{g}_{2}-\frac{\hat{v}_{2}}{R_{2}},
\end{gathered}
$$

where $\hat{g}_{1}$ and $\hat{g}_{2}$ are the small-signal irradiations, $K_{g 1}$ and $K_{g 2}$ are the coefficients of the PV current variation with the irradiation, and $R_{I}$ and $R_{2}$ are the dynamic resistances of the arrays. The dynamic resistance is related to the slope of the $I$ $V$ curve and represents the PV array nonlinear behavior. In the constant current region, it reaches high values, while in the constant voltage region, it has low values.

Introducing (10) and (11) into (7)-(9), reordering and applying Laplace transforms leads to

$$
s \cdot X=A \cdot X+B \cdot U,
$$

where

$$
\begin{gathered}
X=\left[\begin{array}{lll}
\hat{v}_{1} & \hat{v}_{2} & \hat{i}_{L}
\end{array}\right]^{T} \\
U=\left[\begin{array}{llll}
\hat{g}_{1} & \hat{g}_{2} & \hat{d} & \hat{v}_{o}
\end{array}\right]^{T}
\end{gathered}
$$

$$
\begin{gathered}
A=\left[\begin{array}{ccc}
-1 / R_{1} C_{1} & 0 & -D / C_{1} \\
0 & -1 / R_{2} C_{2} & -(1-D) / C_{2} \\
D / L & (1-D) / L & -r_{L} / L
\end{array}\right] \\
B=\left[\begin{array}{cccc}
K_{g 1} / C_{1} & 0 & -I_{L} / C_{1} & 0 \\
0 & K_{g 2} / C_{2} & I_{L} / C_{2} & 0 \\
0 & 0 & \left(V_{1}-V_{2}\right) / L & -1 / L
\end{array}\right] .
\end{gathered}
$$

\section{Voltage Regulation}

\section{A. Plant for the PV1 Voltage Regulation}

The PV1 voltage is regulated by means of the TIBuck switch duty cycle through a single feedback loop. The loop for the PV1 voltage regulation is shown in Fig. 3, where $C_{v 1}$ represents the controller, $S_{v 1}$ the digital sampler, $G_{v 1-d}$ the duty cycle to PV1 voltage transfer function, and $H_{v l}$ the PV1 voltage sensing.

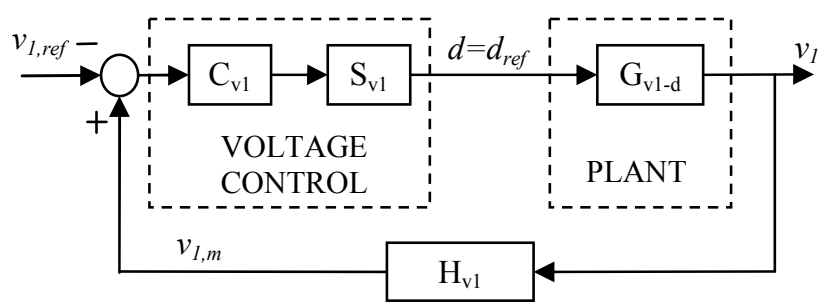

Figure 3. PV1 voltage control loop.

In order to design the controller, the system plant must be worked out. Transfer function $G_{v l-d}$ can be obtained from (12) $-(16)$ and its expression is as follows:

$$
G_{v 1-d}=\frac{\hat{v}_{1}}{\hat{d}}=-\frac{a_{2} \cdot s^{2}+a_{1} \cdot s+a_{0}}{b_{3} \cdot s^{3}+b_{2} \cdot s^{2}+b_{1} \cdot s+b_{0}}
$$

where

$$
\begin{gathered}
a_{2}=I_{L} \cdot L \cdot C_{2} \\
a_{1}=I_{L} \cdot L / R_{2}+I_{L} \cdot r_{L} \cdot C_{2}+D \cdot\left(V_{1}-V_{2}\right) \cdot C_{2} \\
a_{0}=I_{L} \cdot r_{L} / R_{2}+I_{L} \cdot(1-D)+D \cdot\left(V_{1}-V_{2}\right) / R_{2} \\
b_{3}=L \cdot C_{1} \cdot C_{2} \\
b_{2}=L \cdot\left(C_{1} / R_{2}+C_{2} / R_{1}\right)+r_{L} \cdot C_{1} \cdot C_{2} \\
b_{1}\left(R_{1} \cdot R_{2}\right)+r_{L}\left(C_{1} / R_{2}+C_{2} / R_{1}\right)+(1-D)^{2} C_{1}+D^{2} C_{2} \\
b_{0}=r_{L} /\left(R_{1} \cdot R_{2}\right)+(1-D)^{2} / R_{1}+D^{2} / R_{2} .
\end{gathered}
$$

As it can be observed in (17)-(24), the plant zeros and poles are variable depending on the operation point because the converter and the PV arrays are nonlinear. As it has been proved in some papers, the variability of the dynamic resistance diminishes the voltage regulation performance and can compromise the stability for some operation points [5], 
[8]-[10]. For the proposed configuration, the analysis becomes even more delicate because not only one but two different dynamic resistances take part in the control.

The effect of the two dynamic resistances, $R_{1}$ and $R_{2}$, will be analyzed here. In order to ensure stability, the dynamic resistance variation within the whole operation range must be taken into account. For MPP, dynamic resistance can be readily obtained as $R_{M P P}=V_{M P P} / I_{M P P}$, which leads to $R_{M P P I}=11.2 \Omega$ and $R_{M P P 2}=8 \Omega$ in this case [13]. During the system startup or PV power limitation, the system operates in the constant voltage region. At open-circuit voltage, the dynamic resistance has its smallest value, which can be roughly estimated as $R_{\text {min }}=R_{M P P} / 10$. On the other hand, transients can make the system operate at the constant current region, where the dynamic resistance increases very quickly. As a result, $R_{\max }=10 \cdot R_{M P P}$ is used as a rough estimation. The operation range $R_{M P P} / 10<R<10 \cdot R_{M P P}$ must therefore be considered. More details about the dynamic resistance variation range can be consulted in [5].

Fig. 4 shows the bode plot of the transfer function $-G_{v 1-d}$ for the nine combinations of $R_{\min 1}, R_{M P P 1}, R_{\max 1}$ with $R_{\min 2}$, $R_{M P P 2}, R_{\max 2}$. The large influence of the dynamic resistances on the plant can be observed, especially for low frequencies. Two conjugate poles appear between 14000-20000 rad/s (about 2200-3200 Hz), being less damped for high dynamic resistance values. Then, from a certain frequency, all curves tend to join together and the dynamic resistance effect disappears.

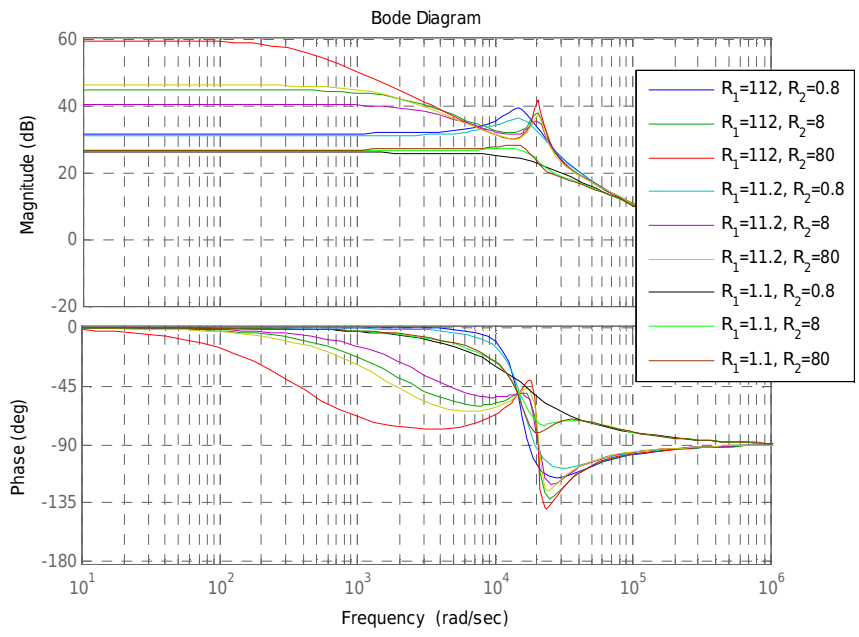

Figure 4. Bode plot of $-G_{v l-d}$ for different $R_{l}$ and $R_{2}$ values.

\section{B. Controller Design for the PV1 Voltage Regulation}

According to Fig. 4, the frequency from which the dynamic resistance effect is no longer present is too high for practical purposes. This frequency could be reduced by increasing the capacitor and inductor values, making it possible to achieve high dynamics as well as prevent the dynamic resistance effect. However, a considerable increase is required in the passive components, which makes that this solution is not worth the effort.
Instead, a crossover frequency $f_{c}$ below the resonance frequency $f_{r}$ is chosen. For the controller design, the resistance values $R_{I}=R_{\max I}$ and $R_{2}=R_{\max 2}$ are considered since the plant bode plot is more problematic concerning stability. In fact, the resonance peak is higher and the phase is lower than for other resistance combinations (see Fig. 4). In order to prevent the resonance peak from cutting the $0 \mathrm{~dB}$ axis and ensure a certain Gain Margin (GM), the crossover frequency cannot be close to the resonance frequency. It is therefore selected as $f_{c}=500 \mathrm{~Hz}$, while $f_{r}=3200 \mathrm{~Hz}$. A pole at $\omega_{p}=2 \pi \cdot 600 \mathrm{rad} / \mathrm{s}$ is added to the conventional PI controller in order to further enhance the gain margin, and the Phase Margin (PM) is imposed to $45^{\circ}$. The controller $C_{v l}$ is thus a type II amplifier, which has three parameters, $K_{P}, T_{n}$ and $\omega_{f}$, and can be expressed as

$$
C_{v 1}=K_{P} \frac{T_{n} \cdot s+1}{T_{n} \cdot s} \cdot \frac{\omega_{p}}{\omega_{p}+s} .
$$

The bode plot of the compensated system is shown in Fig. 5 for three different dynamic resistance combinations. Transfer functions $S_{v l}$ and $H_{v l}$ are modeled as first order lowpass filters with time constants $\tau_{s}=1.5 \cdot T_{S}=15 \mu \mathrm{s}$ and $\tau_{h}=26.5 \mu \mathrm{s}$, respectively, where $T_{S}$ is the sample time (see Fig. 3). Since the regulator is designed for $R_{I}=R_{\max l}$ and $R_{2}=R_{\max 2}$, it can be observed that the control performance are as desired in that case, that is $f_{c}=500 \mathrm{~Hz}$ and $\mathrm{PM}=45^{\circ}$. Besides, thanks to the controller pole at $\omega_{p}=2 \pi \cdot 600 \mathrm{rad} / \mathrm{s}$, the gain margin is high enough, $\mathrm{GM}=13 \mathrm{~dB}$. However, when the system operates with dynamic resistances different from the design values, the voltage response differs. When both PV arrays are operating at MPP, i.e. $R_{I}=R_{M P P I}$ and $R_{2}=R_{M P P 2}$, it can be seen in the figure how the response becomes slower and more damped, with $f_{c}=320 \mathrm{~Hz}, \mathrm{PM}=105^{\circ}$ and $\mathrm{GM}=19 \mathrm{~dB}$. On the other hand, both PV arrays at opencircuit yield to $R_{1}=R_{\min 1}$ and $R_{2}=R_{\min 2}$. In this case, the effect of the dynamic resistances is enormous, slowing down the response to $f_{c}=21 \mathrm{~Hz}$, with $\mathrm{PM}=101^{\circ}$ and $\mathrm{GM}=32 \mathrm{~dB}$.

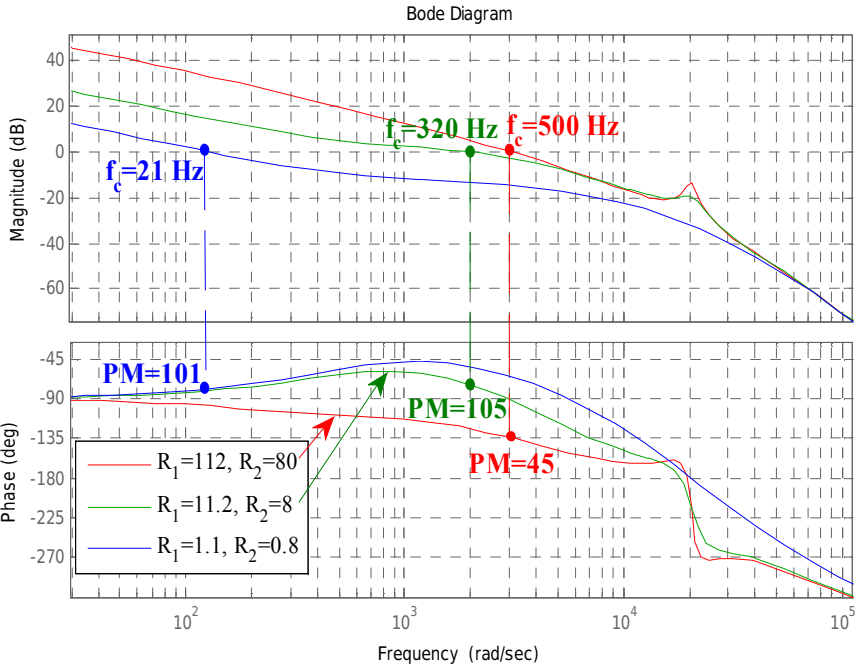

Figure 5. Bode plot of the compensated system $-C_{v l} \cdot S_{v l} \cdot G_{v l-d} \cdot H_{v l}$. 
Fig. 6 shows the effect of the dynamic resistances on the voltage response in more detail. The crossover frequency and the phase margin are represented as a function of $R_{1}$ for three different $\quad R_{2} \quad$ values $\quad\left(R_{\min 2}=0.8 \Omega, \quad R_{M P P 2}=8 \Omega \quad\right.$ and $R_{\max 2}=80 \Omega$ ). It can be clearly observed that, as the dynamic resistances get lower than the maximum values, the phase margin increases. As a consequence, the system is stable for every operation point. Concerning the dynamics, the response slows down when the resistances decrease. However, the voltage response is very quick for every operating point except for the points very close to opencircuit voltage.

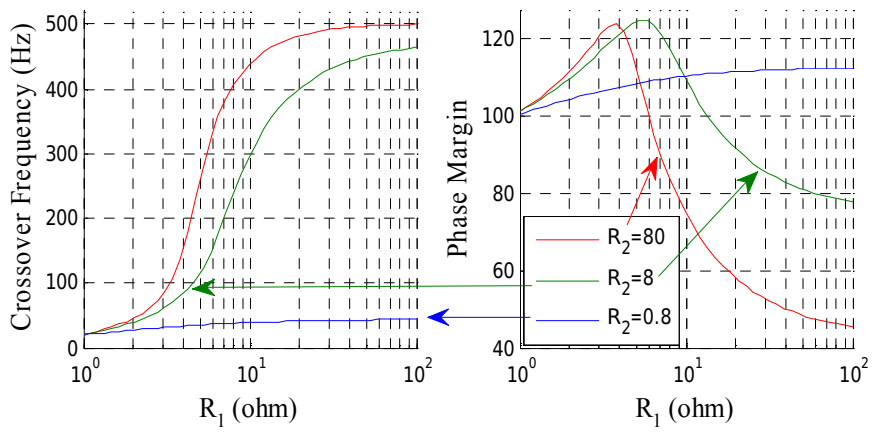

Figure 6. Crossover frequency and phase margin as a function of $R_{1}$ for three different $R_{2}\left(R_{\min 2}=0.8 \Omega, R_{M P P 2}=8 \Omega\right.$ and $\left.R_{\max 2}=80 \Omega\right)$.

\section{PV2 Voltage Regulation}

The PV2 voltage is regulated through a double feedback loop. The outer loop obtains the output voltage reference $v_{0, \text { ref }}$, which is controlled by the boost inverter in the inner loop. The loop for the PV2 voltage regulation is shown in Fig. 7, where $C_{v 2}$ represents the controller, $S_{v 2}$ the digital sampler, $G_{v o, c l}$ the output voltage closed-loop, $G_{v 2-v o}$ the output voltage to PV2 voltage transfer function, and $H_{v 2}$ the PV2 voltage sensing.

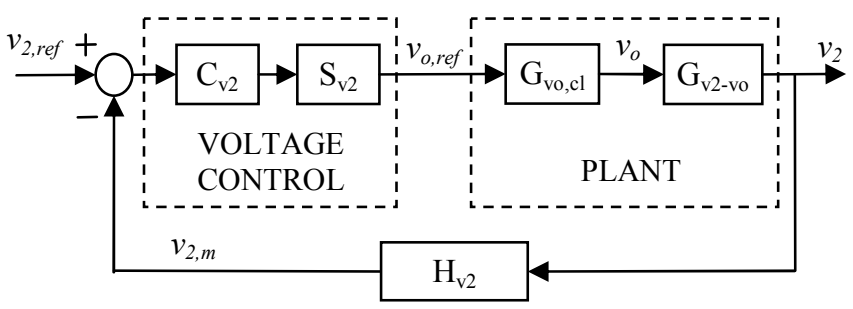

Figure 7. PV2 voltage control loop.

Due to the presence of $100 \mathrm{~Hz}$ ripple in single-phase inverters, the inner loop crossover frequency is $20 \mathrm{~Hz}$. This supposes a dynamic limitation for the PV2 regulation, which is taken into account by means of the closed-loop transfer function $G_{v o, c l}$. In order to obtain the plant transfer function $G_{v 2-v o}$, it can be considered that the PV1 voltage regulation is instantaneous in relation to the PV2 voltage regulation, which makes it possible to remove $R_{I}$ from the plant. Although it is not derived here for space reasons, $G_{v 2-v o}$ can be approximated as a constant value for the frequencies of concern, that is

$$
G_{v 2-v o} \approx \frac{1}{\frac{r_{L}}{R_{2}}+\frac{V_{1}-V_{2}}{R_{2} \cdot I_{L}} \cdot D+(1-D)} .
$$

The controller $C_{v 2}$ is an integral controller, $C_{v 2}=K_{i} / s$, where $K_{i}$ is the integral gain, and is designed to obtain a crossover frequency equal to $10 \mathrm{~Hz}$ for $R_{2} \rightarrow \infty$. However, similarly to the PV1 voltage control, the transfer function $G_{v 2-v o}$ decreases for low values of $R_{2}$, which makes the PV2 voltage response slow down in the constant voltage region.

\section{Simulation Results}

The TIBuck converter, presented in Fig. 1(c) and Table I, and the two PV arrays, shown in Table II, were modeled using the software PSIM.

The PV1 voltage regulation, scheme as shown in Fig. 3, was first validated. For this purpose, the TIBuck output is modeled as a constant voltage source with $V_{o}=40 \mathrm{~V}$. In Fig. 8, the voltage response is represented for an irradiance of $1000 \mathrm{~W} / \mathrm{m}^{2}$ and an array temperature of $25^{\circ} \mathrm{C}$. It consists in $4 \mathrm{~V}$ downward steps of the PV1 voltage reference from $64 \mathrm{~V}$, close to the open-circuit voltage $\left(V_{o c l}=64.8 \mathrm{~V}\right)$, to $48 \mathrm{~V}$, below the MPP voltage $\left(V_{M P P I}=51.9 \mathrm{~V}\right)$. Voltages $v_{l}$, $v_{1, r e f}, v_{2}$, and $v_{o}$ are shown in the figure. It can be observed how PV1 voltage response becomes faster and less damped as PV1 voltage decreases, due to the dynamic resistance $R_{I}$ increase. In any case, the rise time and overshoot are adequate for every operation point.

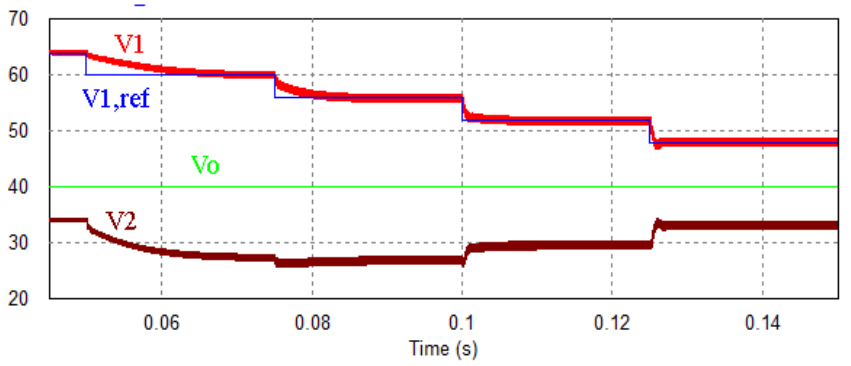

Figure 8. Simulation of the PV1 voltage control.

The regulation of the two PV voltages at the same time was validated in a second simulation. In this case, the output capacitor $C_{0}$ and the boost inverter are replaced by a controlled voltage source, whose value is obtained using $G_{v o, c l}$ transfer function as $v_{o}=G_{v o, c l} \cdot v_{o, r e f}$. In order to regulate PV1 and PV2 voltages, the controls of Fig. 3 and Fig. 7 were applied. In Fig. 9, the voltage response is represented for an irradiance of $1000 \mathrm{~W} / \mathrm{m}^{2}$ and an array temperature of $25^{\circ} \mathrm{C}$. For PV1 voltage, the same downward steps as in Fig. 8 were applied (note that the time scale is different). For PV2 voltage, $2.5 \mathrm{~V}$ reference downward steps were set from $43.5 \mathrm{~V}$, close to the open-circuit voltage $\left(V_{o c 2}=44 \mathrm{~V}\right)$, to $33.5 \mathrm{~V}$, below the MPP voltage $\left(V_{M P P 2}=36 \mathrm{~V}\right)$. The steps are applied at the same time to both voltages, as it would be done by the MPPT algorithm. Voltages $v_{l}, v_{l, r e f}, v_{2}, v_{2, r e f}, v_{o}$, and $v_{o, r e f}$ are shown in the figure. As it can be observed, the response becomes faster for both voltages when the dynamic 
resistances $R_{1}$ and $R_{2}$ increase, as it was predicted. The figure also shows that the PV2 voltage response is affected by the $v_{1, r e f}$ change, which is a disturbance for the control, while the PV1 voltage response is hardly affected by the $v_{2, \text { ref }}$ and consequent $v_{o}$ changes. In any case, a correct regulation of both PV voltages is obtained, which makes the control suitable to maximize the photovoltaic power of two PV arrays at the same time.

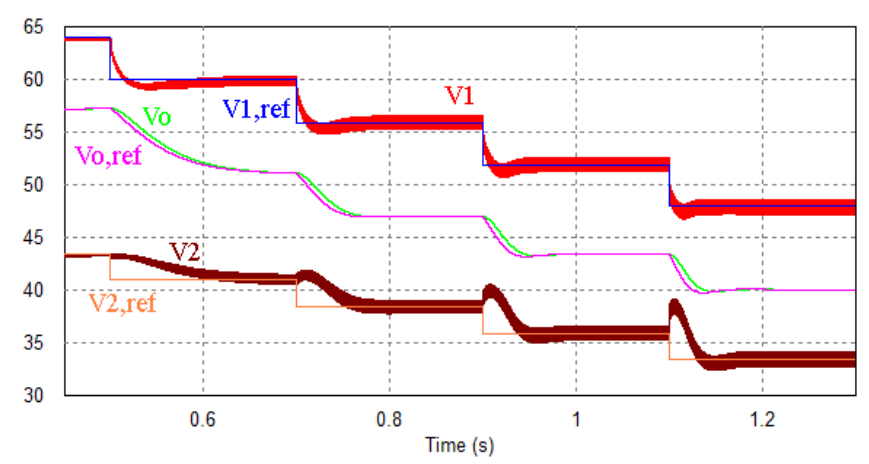

Figure 9. Simulation of the PV1 and PV2 voltage controls.

\section{CONCLUSIONS}

The two-stage inverter composed by a two-input buck converter and a boost inverter shows an interesting solution for low-voltage grid-connected PV systems thanks to its high efficiency and two-MPPT capability with only one extra switch. However, the presence of two nonlinear PV arrays together with the nonlinear converter makes the voltage control design a delicate task.

A control scheme for regulating the two input voltages is first presented in this paper. Then, a system small-signal modeling which accounts for the nonlinear characteristics of the converter and the two PV arrays is derived. Thanks to the derived model, the two controllers are designed and the effect of the dynamic resistances on the control performance is evaluated. Simulation results validated the analysis and showed that the proposed voltage regulation is adequate to perform MPPT of two arrays at the same time.

\section{ACKNOWLEDGMENT}

This work was partially funded by the Public University of Navarre through a doctoral scholarship.

\section{REFERENCES}

[1] REN21 Renewable Energy Policy Network for the $21^{\text {st }}$ Century, "Renewables Global Status Report 2014".

[2] E. Hofreiter and A. M. Bazzi, "Single-state boost inverter reliability in solar photovoltaic applications," in Power and Energy Conference at Illinois (PECI), pp. 1-4, 2012.

[3] W. Zhao, D. D. -C. Lu, and V. G. Agelidis, "Current control of gridconnected boost inverter with zero steady-state error," IEEE Transactions on Power Electronics, vol. 26, no. 10, pp. 2825-2834, 2011

[4] A. Maki, and S. Valkealahti, "Power losses in long string and parallel-connected short strings of series-connected silicon-based photovoltaic modules due to partial shading conditions," IEEE Transactions on Energy Conversion, vol. 27, no. 1, pp 173-183, 2012.

[5] A. Urtasun, P. Sanchis, and L. Marroyo, "Adaptive voltage control of the $\mathrm{dc} / \mathrm{dc}$ boost stage in PV converters with small input capacitors," IEEE Transactions on Power Electronics, vol. 28, no. 11, pp. 50385048,2013

[6] S. V. Dhople, J. L. Ehlmann, A. Davoudi, and P. L. Chapman, "Multiple-input boost converter to minimize power losses due to partial shading in photovoltaic modules," in Energy Conversion Congress and Exposition (ECCE), pp. 2633-2636, 2010.

[7] J. Sebastian, P. Villegas, F. Nuno, and M. Hernando, "Highefficiency and wide-bandwidth performance obtainable from a twoinput buck converter," IEEE Transactions on Power Electronics, vol. 13, no. 4, pp. 706-717, 1998.

[8] M. G. Villalva, T. G. de Siqueira, and E. Ruppert, "Voltage regulation of photovoltaic arrays: Small-signal analysis and control design," IET Power Electronics, vol. 3, no. 6, pp. 869-880, 2010.

[9] L. Nousiainen, J. Pukko, A. Maki, T. Messo, J. Huusari, J. Jokipii, J. Viinamaki, D. T. Lobera, S. Valkealahti, and T. Suntion, "Photovoltaic generator as an input source for power electronic converters," IEEE Transactions on Power Electronics, vol. 28, no. 6, pp. 3028-3038, 2013.

[10] W. Xiao, W. G. Dunford, P. R. Palmer, and A. Capel, "Regulation of photovoltaic voltage," IEEE Transactions on Ind. Electronics, vol. 54, no. 3, pp. 1365-1374, 2007.

[11] C. R. Sullivan, J. J. Awerbuch, and A. M. Latham, "Decrease in photovoltaic power output from ripple: simple general calculation and the effect of partial shading," IEEE Transactions on Power Electronics, vol. 2, no 2, pp. 740-747, 2013.

[12] A. Urtasun, P. Sanchis, and L. Marroyo, "Limiting the power generated by a photovoltaic system," in Multi-Conference on Systems, Signals and Devices (SSD), pp. 1-6, 2013.

[13] A. Safari and S. Mekhilef, "Simulation and hardware implementation of incremental conductance MPPT with direct control method using Cuk converter," IEEE Transactions on Industrial Electronics, vol. 58, no. 4, pp. 1154-1161, 2011. 